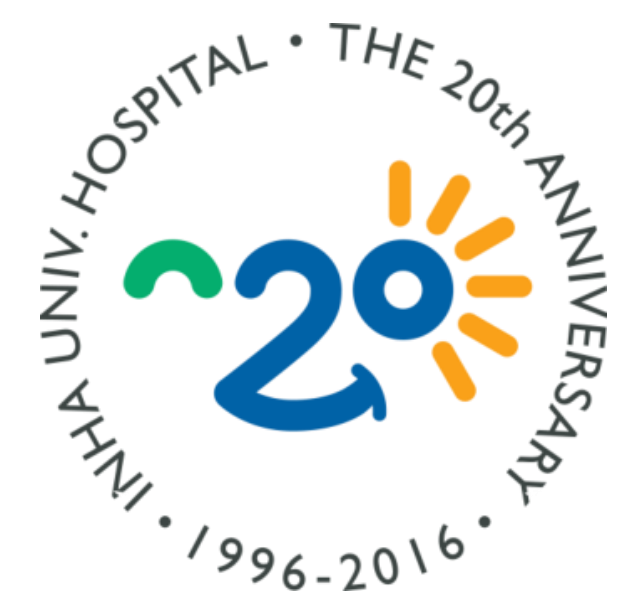

\title{
A comparison of ultrasound-guided interscalene block and combined suprascapular nerve and costoclavicular brachial plexus block for arthroscopic shoulder surgery: a non-inferiority trial
}

Yoon Jung Kim, MDa . Hyunzu Kim, MD, PhDa. Na Eun Kim, MDa. Chunwoo Yang, MDa,.

${ }^{a}$ Department of Anesthesiology and Pain Medicine, Inha University College of Medicine, Incheon, South Korea

\section{Background}

Several diaphragm sparing nerve blocks were evaluated. In this study, we compared ultrasound guided interscalene block(ISB) with combined proximal suprascapular nerve block(SSNB) and costoclavicular block(CCB) for arthroscopic shoulder surgery.

\section{Hypotheses}

We hypothesized that combined SSNB-CCB was non-inferior to ISB in terms of immediate postoperative analgesia for arthroscopic shoulder surgery with a reduced risk of hemidiaphragmatic paralysis(HDP).

\section{Methods}

Adult inpatients $(20-80$ years of age, American Society of Anesthesiologists physical status I - 3) scheduled for arthroscopic shoulder surgery were included. Patients were randomly assigned to receive either ISB (group I) or combined SSNB-CCB (group SC).

- The primary outcome : pain score at $30 \mathrm{~min}$ in the PACU

- The secondary outcomes: pain score $(6 \mathrm{hr}, 24 \mathrm{hr})$, worst pain score(WP), supplemental analgesia (IV morphine equivalents), incidence of HDP, block related outcomes (performance time, patient discomfort, motor block, block duration), adverse events (vascular puncture, LA toxicity, and unintentional paresthesia)

Figure I. . CONSORT E-flow chart

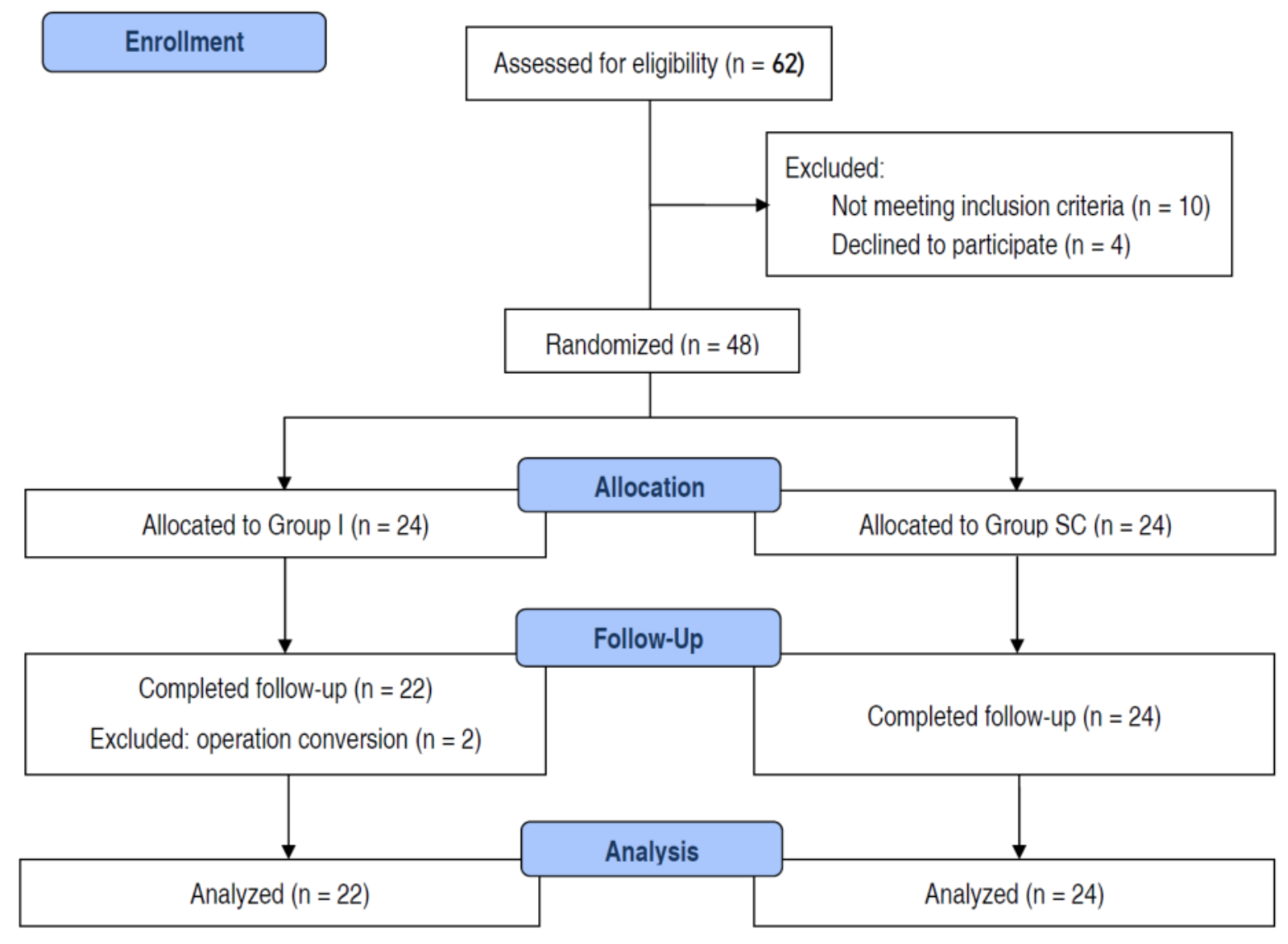

\section{Results}

Group SC was not inferior to the group $I$ in terms of the primary outcome $(P=0.987$, the difference of the medians, $0 ; 95 \% \mathrm{Cl} 0-0)$. The pain scores at other time points were not significantly different between the groups (Fig. 2). Supplemental analgesia was not significantly different in both groups at PACU and $24 \mathrm{hr}$ (Table 2).
Figure 2. Postoperative pain scores

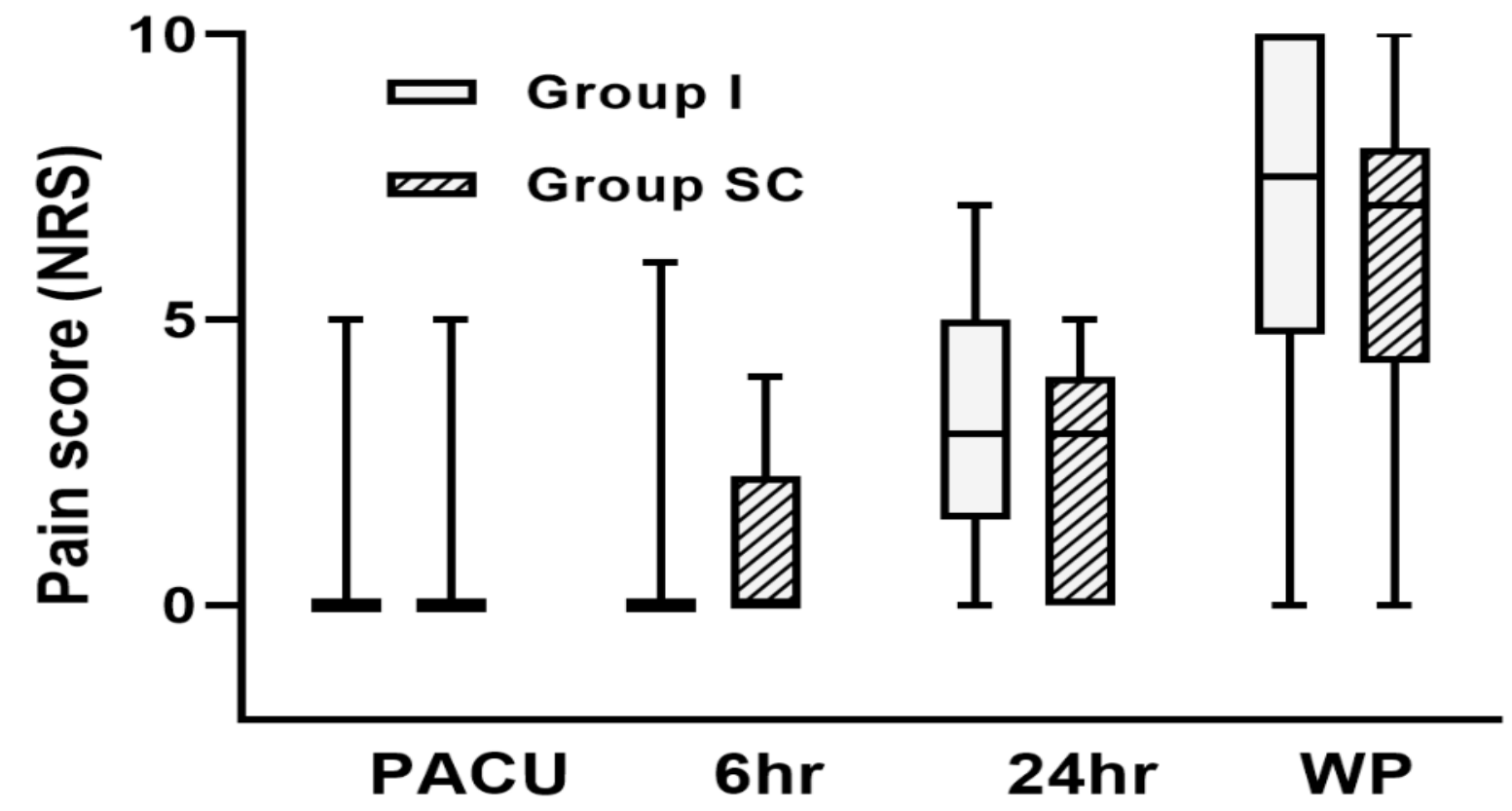

Table I. Supplemental analgesia

\begin{tabular}{lccc}
\hline & Group I $(n=22)$ & Group SC $(n=24)$ & $P$ value \\
at PACU $(\mathrm{mg})$ & $0(0-0)$ & $0(0-0)$ & .344 \\
$24 \mathrm{hr}(\mathrm{mg})$ & $3.3(0-3.3)$ & $3.3(0-6.7)$ & .990
\end{tabular}

Amount of supplemental opioids were expressed as a IV morphine equivalent dose.

Complete or partial HDP occurred in a higher incidence in group I than group SC $(73 \%$ vs $8 \% ; \mathrm{P}<0.00 \mathrm{I})$.

Block-procedure time was longer in combined block compared to ISB.

Other secondary outcomes were comparable in both groups(Table 3).

Table2. Block performance characteristics

\begin{tabular}{lccc}
\hline & Group I $(\mathrm{n}=22)$ & Group SC $(\mathrm{n}=24)$ & $P$ value \\
Procedure time (min) & $2.3(\mathrm{I} .0)$ & $5.9(3.0)$ & $<0.00 \mathrm{I}$ \\
Patient discomfort (NRS) & $3(2-3)$ & $2(1-3)$ & 0.417 \\
Paresthesia (n) & 2 & 1 & 0.497 \\
Block duration (hr) & $14(10-16)$ & $14(1 \mathrm{I}-19)$ & 0.627 \\
Patient satisfaction (NRS) & $10(8-10)$ & $10(10-10)$ & 0.156 \\
\hline
\end{tabular}

Table 3. Block related adverse events

\begin{tabular}{lccc}
\hline & Group I $(n=22)$ & Group SC $(n=24)$ & $P$ value \\
Dyspnea $(n)$ & 1 & 1 & .733 \\
at PACU & 0 & 0 & \\
$24 \mathrm{hr}$ & & & \\
Handgrip weakness $(\mathrm{n})$ & & & \\
(no/partial/complete) & $0 / 1 \mathrm{l} / 10$ & $0 / 14 / 10$ & .657 \\
at PACU & $20 / 2 / 0$ & $22 / 2 / 0$ & .407 \\
$24 \mathrm{hr}$ & & & \\
Paresthesia (n) & 2 & 0 & .223 \\
at PACU & 6 & 9 & .337 \\
$24 \mathrm{hr}$ & & & \\
\hline
\end{tabular}

\section{Discussion}

This study shows that combined SSNB-CCB was non-inferior to ISB in terms of pain score at $30 \mathrm{~min}$ in PACU, the primary outcome, and subsequent pain scores were similar in both groups. The overall incidence of HDP with combined block was lower than that with ISB. 